\title{
Yes, science is costly indeed
}

\author{
Tam-Tri Le \\ Centre for Interdisciplinary Social Research \\ Phenikaa University, Hanoi, Vietnam
}

2021 December 28

Science is costly. The public can see this. Sometimes people also complain about how costly science is (the old legends about mad scientists wasting all your tax money, you know). But are such huge costs justified?

I have just read an article where the author stated (and expressed her genuine emotions about) various aspects of how science costs so much (Arrowsmith, 2020). It is in every little (as well as big) detail, such as instruments, facilities (and their staff), salaries for scientists, safety measures, and a lot of other obvious but often forgotten stuff that make up the working environment as it is. That was a story from the United States of America. However, similar patterns about how science may be viewed as "irrationally costly" were also found present in developing countries like Vietnam, where funding is comparatively much more limited (Vuong, 2018).

There are a lot of hidden costs and values taken for granted, particularly intellectual sharing and established background mindsets in social systems. They are "hidden" because most people are not aware of their values. Well, values are subjective, and people all have their own ways of evaluating the perceived worth of science. And if people are not even aware of the existence of these values, then it is more likely for them to deem science as "overpriced".

But are the values contributed by science actually related to your everyday life? The one firmly answering "No" must have just visited human civilization for the very first time.

There is also one more interesting thing I found from Arrowsmith's paper about the cost of science: the mindset behind the criticisms in relation to environmental issues. There are people who think that science is wasteful for conducting research on "faraway" topics - typically nature protection. It is almost the year 2022 and surely I do not have to explain how ridiculous that view is. It is clearly the problem of collective mindset and subjective value perception (Vuong, 2021).

Anyway, rest assured that we scientists are the ones who are most concerned about the high costs of doing science. For example, apparently, my colleagues and I do sweat a lot each time we look at the price tags of article processing charges (APCs) in relation to our wallets. 


\section{References}

Arrowsmith, S. (2020). The Real Cost of Science. Swette Center for Environmental Biotechnology. http://environmentalbiotechnology.org/2020/03/18/the-real-cost-ofscience/

Vuong, Q.-H. (2018). The (ir)rational consideration of the cost of science in transition economies. Nature Human Behaviour, 2(1), 5. https://doi.org/10.1038/s41562-017-02814

Vuong, Q.-H. (2021). The semiconducting principle of monetary and environmental values exchange. Economics and Business Letters, 10(3), 284-290.

https://doi.org/10.17811/ebl.10.3.2021.284-290 